\title{
Detrended cross-correlation analysis of epilepsy electroencephalagram singals
}

\author{
Junchang Zhao ${ }^{\mathrm{a}}$ Wanhu Dou $^{\mathrm{b}}{\text { Hongda } \mathrm{Ji}^{\mathrm{c}} \text { Jun Wang }}^{\mathrm{d}}$ \\ Image Processing and Image Communications Key Lab, College of Geo \& Bio Information, Nanjing \\ Univ of Posts \& Telecomm, Nanjing 210003, China \\ aemail: 1096134626@qq.com, bemail: 774660336@qq.com, cemail: 1345267276@qq.com, \\ demail: wangj@njupt.edu.cn
}

Key words: detrended cross-correlation analysis, epilepsy, electroencephalagram

\begin{abstract}
The cross-correlation performance between epilepsy electroencephalogram (EEG) signals reflects the status of epilepsy patients which has importance for analyzing long-range correlation of non-stationary signals. For the first time, detrended cross-correlation analysis (DCCA) was applied to analyze different physiological and pathological states of epilepsy EEG signals. It were compared the difference of DCCA values between epilepsy patients' EEG signals and normal subjects' EEG signals. It was found that the DCCA values of epilepsy patients' EEG signals increased compared the normal subjects' EEG signals which can be helpful for medical diagnosis and treatment.
\end{abstract}

\section{Introduction}

In some cases, the different signals will exhibit a cross-correlation relationship. The presence of these features between signals may be utilized in real life to conduct various analyzes. In the earthquake, the cross-correlation between the degree of noise signals with different detector array antenna can be used as a predictive signal of earthquakes and volcanic eruptions [1]. In business, assets and investment risk estimates are based on the cross-correlation matrix for analysis [2]. In quantum information processing nanotechnology, electronic correlation promoted calculating the cross correlation between the current noise, and were compared to the standard device, and this noise characteristics of the signal can be determined whether it will be inverted [3].

In medicine, it can also be used for diagnosis and treatment of the cross-correlation characteristics between the signals of different physiological and pathological signals. The paper firstly applied detrende cross-correlation analysis (DCCA) to analyze epilepsy signals, i.e., used least squares curve fitting analysis and detrended covariance to process epilepsy electroencephalogram (EEG) data[4-6]. The DCCA value differences exist between different patients could be shown from the computed results which could guide rehabilitation assessment and treatment. In the same time, it also could contribute to the clinical diagnosis, therapeutic evaluation and study of the mechanism of injury.

Epilepsy is a group of diseases and syndromes, i.e., it is brain sudden abnormal discharge which resulting in a chronic disease of the transient brain dysfunction. Abnormal discharge of neurons in the parts and different range of discharge diffusion can be expressed as the onset of movement, feeling the autonomic, consciousness and mental disorders. It is one of the common clinical symptoms caused by a variety of reasons. The human nervous system has complex excited inhibit the feedback mechanism, in order to maintain the stability of the neuronal membrane. Epilepsy is the enhancement of the excitatory process and weakening of the inhibitory process. Therefore, the analysis of epileptic EEG helps for clinical diagnostics and medical research.

For the first time, detrended cross-correlation analysis (DCCA) was applied to analyze different physiological and pathological states of epilepsy EEG signals. It were compared the difference of DCCA values between epilepsy patients' EEG signals and normal subjects' EEG signals. It was found that the DCCA values of epilepsy patients' EEG signals increased compared the normal subjects’ EEG signals which can be helpful for medical diagnosis and treatment. 


\section{Detrended Cross-Correlation Analysis}

In order to overcome the highly non-stationary time series, an improved method of analysis of covariance (detrended cross-correlation analysis, DCCA) for cross-correlation analysis of biomedical data.

DCCA is a method based on the detrended covariance analysis. For non-stationary signals, least squares linear fit was made and the trend was eliminated, it can find the cross-correlation between two non-stationary time series.

DCCA provides clinicians with a method studying the long correlation which was generated by internal mechanism of physiological signals. The study did not consider the stimulus produced by external system which had no relationship with the system itself. The method used the entire sequence to calculate. So it can provide useful information which can be helpful for doctor diagnosis of a disease.

DCCA was applies into epilepsy patients’ and healthy subjects’ EEG signals whose results can be helpful for doctor diagnosis of a disease.

Provided that two time series $\left\{y_{i}\right\}$ and $\left\{y_{i}\right\}, i=1,2, \ldots, N$.

It was defined that,

and

$$
R_{k}=y_{1}+y_{2}+\ldots+y_{k}
$$

$$
R_{k}^{\prime}=y_{1}^{\prime}+y_{2}^{\prime}+\ldots+y_{k}^{\prime}
$$

Where $k \leq N$.

The mean value were: ${ }_{\mu}=\overline{y_{i}}=(1 / N) \sum_{i=1}^{N} y_{i}^{\prime} \mu^{\prime}=\overline{y_{i}^{\prime}}=(1 / N) \sum_{i=1}^{N} y_{i_{i}^{\prime}}$.

The variance were: $\sigma^{2}=\overline{\left(y_{i}-\overline{y_{i}}\right)^{2}}, \sigma^{\prime 2}=\overline{\left(y^{\prime}-\overline{y_{i}^{\prime}}\right)^{2}}$.

Assuming the cross-correlation function were : $A(n)=\overline{\left(y_{k}-\mu\right)\left(y_{k+n}-\mu\right)} / \sigma^{2}$ and

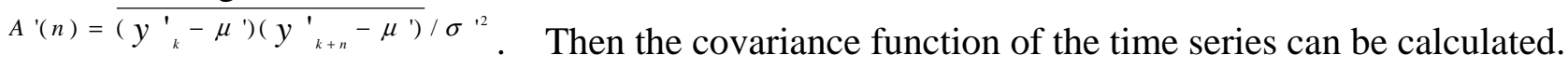

The time sequence was divided into $N-n$ overlaped segments whose each section containing $n+1$ data, and then each segment was performing a least squares linear fit that was: $\widetilde{R}_{k, i}$ and $\widetilde{R}_{k, i}^{\prime}$.

Each segment's covariance was calculated:

$$
f_{\text {DCCA }}^{2}(n, i)=1 /(n-1) \sum_{k=i}^{i+n}\left(R_{k}-\widetilde{R}_{k, i}^{\prime}\right)\left(R_{k}^{\prime}-\widetilde{R}_{k, i}^{\prime}\right)
$$

At last, the whole time series's covariance was:

$$
F_{D C C A}^{2}(n)=(N-n)^{-1} \sum_{i=1}^{N-n} f_{D C C A}^{2}(n, i)
$$

The above calculation was repeated on segments and then it can be drawn the curve of $F$ (n) and segment size $n$. The slope of $\log (F(N))$ vs $\log (n)$ determined the scaling exponent (self-similarity parameter) $\alpha$ [4-6].

\section{Data Analysis}

During the numerical calculation, the EEG were taken from healthy normal people, and people suffering from epilepsy. The two kinds EEG data was analyzed and the results were shown in Table 1 respectively. As an example, the DCCA of two EEG data taken from healthy normal people, and people suffering from epilepsy were shown in Figure 1.

Table 1 DCCA of two kinds of subjects'EEG

\begin{tabular}{lcc}
\hline Subjects & Helthy subjects & Epilepsy subjects \\
1 & 1.035453 & 1.185193 \\
2 & 0.861638 & 1.239772 \\
3 & 1.13327 & 1.520877 \\
4 & 1.187523 & 1.294416 \\
\hline
\end{tabular}




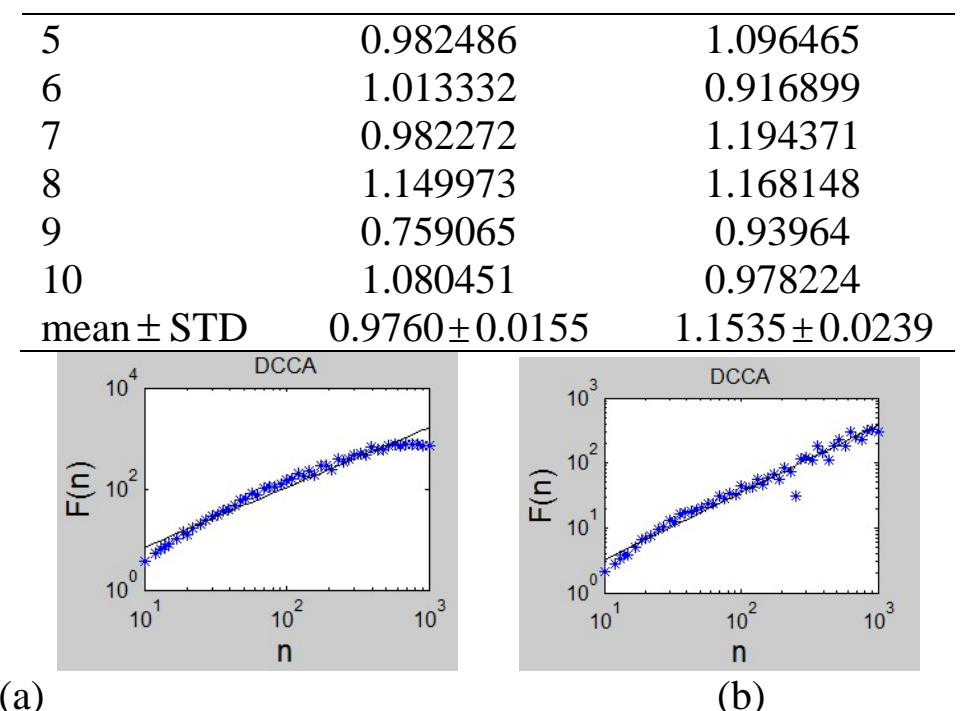

(a)

(b)

Figure 1 DCCA of two kind of EEG

(a) DCCA of epilepsy patient's EEG, (b)DCCA of a helthy subject's EEG

It can be seen from the numerical results as shown in Table 1 that the average DCCA value of epilepsy patients' EEG is greater than that of the normal subjects' EEG. It is particularly important to help clinical diagnosis.

Fluctuation range showed the self-similar characteristics become unstable when human brain EEG status developing from normal to epilepsy status.

After verification of large amounts of data, it can be achieved a helpful diagnostic method for to make difference between healthy subjects’ and epilepsy subjects’ EEG.

\section{Conclusions}

The paper appllied DCCA to analyze the EEG of healthy subjects and epilepsy subjects. It was found that the cross-correlation exists in normal subjects' EEG and the cross-correlation will decrease for the epilepsy patients' EEG. In clinical applications, doctors can check the DCCA value if it was in the normal EEG' DCCA range to determine if the epilepsy will occur.

\section{Acknowledgements}

This work was supported by the National Natural Science Foundation of China (Grant Nos. 61271082, 61201029, 61102094), the Natural Science Foundation of Jiangsu Province (Grant Nos. BK2011759, BK2011565) and Foundation of Nanjing University of Posts and Telecommunications (JG03212JX02, JG03210JX19, 2011XSG11).

\section{References}

[1] M.Campillo, A.Paul, Long-range correlations in the diffuse seismic coda, Science, 299 (2003) 547-549.

[2] L.Laloux, P. Cizeau, J.-P. Bouchaud, M. Potters, Phys. Rev. Lett. 83 (1999) 1467-1470.

[3] P.Samuelsson, E.V. Sukhorukov, M. Buttiker, Orbital entanglement and violation of bell inequalities in mesoscopic conductors, Phys. Rev. Lett. 91(2003) 157002.

[4] B. Podobnik, H.E. Stanley.Detrended cross-correlation analysis: A new method for analyzing two nonstationary time series, Phys. Rev. Lett. 100 (2008) 084102.

[5] W.-X. Zhou. Multifractal detrended cross-correlation analysis for two nonstationary signals. Phys. Rev. E 77 (2008) 066211.

[6] P.-A. Absil, R. Sepulchre, A. Bilge, P. Gerard, Nonlinear analysis of cardiac rhythm fluctuations using DFA method, Physica A 272 (1999) 235-24 